\title{
"Stimulateur cardiaque CRT» en cas de défaillance du muscle cardiaque
}

\section{Swiss Medical Board}

\author{
Après une étude approfondie de la littérature, le Swiss Medical Board (SMB) a conclu \\ que la pose d'un stimulateur cardiaque CRT, comme complément à un traitement \\ par médicaments, pouvait être appropriée chez des patients souffrant d'insuffi- \\ sance cardiaque sévère.
}

On parle d'insuffisance cardiaque lorsque le muscle cardiaque ne peut plus assurer un débit sanguin suffisant pour satisfaire les besoins de l'organisme. Dans sa forme sévère, la maladie est très éprouvante pour le patient et conduit finalement à sa mort.

En Suisse, plus de 150000 personnes souffrent d'insuffisance cardiaque. Au-delà du traitement médicamenteux, il est possible, pour un petit groupe de ces patients, de renforcer la capacité de pompage du cœur avec un stimulateur cardiaque spécifique (la thérapie dite de resynchronisation cardiaque, en anglais "cardiac resynchronisation therapy" respectivement «CRT»).

Le présent rapport vérifie si, comparé à d'autres procédés thérapeutiques, un traitement par stimulateur cardiaque CRT offre aux patients concernés un avantage appréciable en termes de survie et de qualité de vie. Sur la base de la littérature étudiée, les patients ont été répartis dans quatre groupes selon le niveau de gravité

\section{Post Publication Review}

Des experts ont d'ores et déjà participé à la formulation des présentes directives; néanmoins tous les partenaires intéressés sont explicitement invités à prendre position dans le cadre d'une Post Publication Review (PPR). Les processus en vigueur jusqu'en 2014 ont été appliqués au présent rapport. Depuis 2014, les partenaires concernés sont impliqués dans des hearings à divers niveaux du processus. Les personnes intéressées sont priées de nous soumettre leurs commentaires concernant le rapport "Stimulateur cardiaque CRT» jusqu'au 15 septembre 2015. Les rapports contenant les résultats de la PPR et une analyse seront disponibles sur le site Internet du SMB. de leur maladie. Chez les patients des deux groupes avec une insuffisance cardiaque sévère, la thérapie CRT a réduit la mortalité et le taux d'hospitalisation et amélioré la fonction cardiaque. Pour ces groupes de patients, le rapport efficacité-coûts est favorable.

Pour ces groupes de patients, le rapport efficacité-coûts est favorable.

Après l'évaluation des résultats de l'analyse, le Conseil d'experts a formulé les recommandations suivantes:

- Le traitement par stimulateur cardiaque CRT des patients souffrant d'insuffisance cardiaque sévère est fondamentalement pertinent.

- La plupart des patients de ce groupe sont d'un âge avancé et présentent d'autres maladies graves qui entravent également la qualité de vie. Il est d'autant plus important

- que les médecins traitants, notamment le médecin de famille et le cardiologue, procèdent à des investigations minutieuses et se concertent et

- que le patient respectivement son représentant bénéficie d'une information complète, concernant en particulier l'éventualité d'une prolongation de ses souffrances.

- Pour les patients souffrant d'une insuffisance cardiaque légère, la thérapie par stimulateur cardiaque CRT n'est, en principe, pas recommandée.

Le rapport complet ainsi que des informations complémentaires concernant le Swiss Medical Board peuvent être consultés sur le site www.swissmedicalboard.ch. 